A manuscript submitted to

IEEE Transactions on Wireless Communications

as a letter

\title{
The Effect of NBI on UWB Time-Hopping Systems ${ }^{1}$
}

\author{
Xiaoli Chu and Ross D. Murch ${ }^{2}$, Senior Member, IEEE \\ Center for Wireless Information Technology \\ Department of Electrical \& Electronic Engineering \\ The Hong Kong University of Science \& Technology \\ Clear Water Bay, Kowloon, Hong Kong \\ vivichu@ust.hk, eermurch@ee.ust.hk
}

September 18, 2002

\begin{abstract}
This letter presents an analysis of the effect of narrowband interference (NBI) on ultra-wideband (UWB) time-hopping (TH) systems in the presence of multipath fading using both analytical derivations and simulations. Our analysis demonstrates that NBI may be an issue in some instances. In addition we suggest three NBI suppression schemes for combating NBI in UWB TH systems. Single-link performance of these schemes in conjunction with a Rake-type receiver structure is estimated for both the ideal All-Rake receiver and the simpler Partial-Rake receiver in an indoor environment. Two UWB pulse shapes that meet the FCC rules for UWB communications are considered in the investigation.
\end{abstract}

Index Terms - ultra-wideband, time hopping, BPPM, narrowband interference, Rake receiver, MMSE.

\footnotetext{
${ }^{1}$ Supported by the Hong Kong Research Grant Council.

${ }^{2}$ Corresponding Author. Phone: (+852) 2358 7044. Fax: (+852) 23351834.
} 


\section{INTRODUCTION}

Ultra-wideband (UWB) time-hopping (TH) systems communicate with carrierless time-hopping subnanosecond pulses [1]. The transmitted signal occupies an ultra wide bandwidth of up to a few gigahertz, which allows a low power spectral density and enables UWB TH systems to coexist with conventional communications systems. The FCC allows UWB communication devices to operate under Part 15 rules if they are restricted to the spectrum $3.1-10.6 \mathrm{GHz}$ with a power spectral density of less than $-41.3 \mathrm{dBm} / \mathrm{MHz}$ [8]. UWB TH systems have inherent immunity to narrowband interference (NBI) because of the very short time windowing at the receiver and the high correlation of narrowband signals over a short time interval. However, due to the low power spectral density, it is expected that even this interference immunity is not sufficient to suppress high levels of NBI, e.g., an IEEE 802.11a WLAN device operating nearby (which would be seen as NBI by typical UWB systems). The UWB $\mathrm{TH}$ receiver may be jammed if such interference is not properly suppressed.

In this letter we present an analysis of the effect of NBI on a victim UWB TH receiver in an indoor multipath environment. Two pulse shapes that meet the FCC UWB communications rules [8] are proposed and used. In addition we investigate three NBI suppression schemes that are designed to operate in conjunction with a conventional Rake-type UWB receiver: 1) a scheme exploiting the property of the time-domain modulation parameter, 2) frequency-domain notch filtering, and 3) Minimum Mean-Square-Error Combining (MMSEC).

Related published research includes [2], [3] where NBI was modeled as a zero-mean random process while the effect of NBI to UWB systems was studied in AWGN channels [3]-[5]. Our work is different in that we consider the multipath effect of UWB indoor transmission [6] and provide performance evaluation for two types of Rake structure introduced in [7]. This includes an All-Rake (ARake) and a simpler Partial-Rake (PRake) that combines the $L_{p}$ first arriving paths. We also utilize pulse shapes that meet Part 15 UWB rules and consider various forms of NBI including sinusoidal signals, band-limited signals, and OFDM-based WLAN signals. 


\section{UWB TIME-HOPPING SYSTEM MODEL}

\section{A. Time-Hopping Format}

A typical TH format with binary pulse-position modulation (BPPM) [1] is given by:

$$
\mathrm{s}_{t}(t)=\sum_{i=0}^{N_{s}-1} w_{t}\left(t-i T_{f}-c_{i} T_{c}-\delta d\right)
$$

where, $T_{f}$ is the pulse repetition time, $w_{t}(t)$ represents the transmitted pulse, $\left\{c_{i}\right\}$ is a distinctive time-hopping sequence, $T_{c}$ is the time hopping unit, $d$ represents a binary ( 0 or 1$)$ symbol, the modulation parameter $\delta$ is on the order of the pulse width $T_{w}$, and $N_{s}$ pulses are transmitted per symbol interval. For a fixed $T_{f}$, the symbol rate $R_{s}$ determines the number $N_{s}$ via $R_{s}=1 /\left(N_{s} T_{f}\right) \mathrm{sec}^{-1}$.

\section{B. The Channel Model \& Received Signal}

We consider a single-link UWB system in a UWB indoor channel [6], which is defined by its power delay profile (PDP):

$$
g(t)=\sum_{k=1}^{N_{r}}\left|\beta_{k}\right|^{2} \delta\left(t-\tau_{k}\right)
$$

where $\beta_{k}$ represents the complex-valued amplitude of the $k^{\text {th }}$ path, the phase of $\beta_{k}$ is modeled as a random variable uniformly distributed in $[0,2 \pi]$, the power gains $\left|\beta_{k}\right|^{2}$ are generated by a superposition of large and small-scale statistics, which are modeled as lognormal distribution and Gamma distribution respectively [6], $N_{r}$ denotes the number of propagation paths arriving to the receiver, $\delta(\cdot)$ is Kronecker Delta, and $\tau_{k}$ is the excess delay of the $k^{\text {th }}$ path. Both $\left\{\beta_{k}\right\}$ and $\left\{\tau_{k}\right\}$ are assumed to be known to the receiver.

The composite received signal (over a symbol interval of $0 \leq t \leq N_{s} T_{f}$ ) at the output of the receiver's antenna is expressed as

$$
\mathrm{r}(t)=\sum_{k=1}^{N_{r}} \beta_{k} \sum_{i=0}^{N_{s}-1} A w_{r}\left(t-i T_{f}-c_{i} T_{c}-\delta d-\tau_{k}\right)+\mathrm{i}(t)+\mathrm{n}(t)
$$

where, $A$ denotes the amplitude of the received pulse $w_{r}(t), \mathrm{i}(t)$ represents NBI, and $\mathrm{n}(t)$ is the zero-mean AWGN. It is assumed that $T_{f}$ is larger than the maximum excess delay and no multipath interference exists.

\section{Correlation Rake Reception and Combining}

Our work considers two types of Rake structure [7]: the All-Rake (ARake) that combines all the resolvable paths and the simpler Partial-Rake (PRake) that combines 
the $L_{p}$ first arriving paths of the resolvable multipath components. In the theoretical analysis herein we assume an ARake receiver, which consists of multiple correlators employing the template signal of $v(t)=w_{r}(t)-w_{r}(t-\delta)$ [1], followed by a linear combiner. When appropriately synchronized and assuming the pulse shape $w_{r}(t)$ known to the receiver, the correlation output over a symbol interval of the $k^{\text {th }}$ path, denoted by $\mathrm{r}_{k}\left(k=1,2, \ldots, N_{r}\right)$, is given by:

$$
\mathrm{r}_{k}=\sum_{i=0}^{N_{s}-1} \int_{\tau_{k}+i T_{f}}^{\tau_{k}+(i+1) T_{f}} \mathrm{r}(t) v\left(t-i T_{f}-c_{i} T_{c}-\tau_{k}\right) d t=(1-2 d) \mathrm{s}_{k}+\mathrm{i}_{k}+\mathrm{n}_{k}
$$

where,

$$
\begin{aligned}
\mathrm{s}_{k} & =\beta_{k} A N_{s} \int_{-\infty}^{\infty} w_{r}(t)\left[w_{r}(t)-w_{r}(t-\delta)\right] d t=\beta_{k} A N_{s} m_{p}(\delta) \\
\mathrm{i}_{k} & =\sum_{i=0}^{N_{s}-1} \int_{-\infty}^{\infty} \mathrm{i}\left(t+i T_{f}+c_{i} T_{c}+\tau_{k}\right) v(t) d t \\
\mathrm{n}_{k} & =\sum_{i=0}^{N_{s}-1} \int_{-\infty}^{\infty} \mathrm{n}\left(t+i T_{f}+c_{i} T_{c}+\tau_{k}\right) v(t) d t=\sum_{i=0}^{N_{s}-1} \mathrm{n}_{i, k}
\end{aligned}
$$

Each test statistics $\mathrm{r}_{k}$ is the sum of $N_{s}$ correlations of the template signal $v(t)$ at different time shifts with the received signal $\mathrm{r}(t)$. It is assumed that the sampled noise $\mathrm{n}_{i, k} \sim \mathrm{N}\left(0, \sigma_{r}^{2}\right)$, then $\mathrm{n}_{k} \sim \mathrm{N}\left(0, N_{s} \sigma_{r}^{2}\right)$.

For simplicity of notation, we stack these $\mathrm{r}_{k}$ 's in a $N_{r} \times 1$ vector $\mathbf{r}$, so that:

$$
\mathbf{r}=(1-2 d) \mathbf{s}+\mathbf{i}+\mathbf{n}
$$

Then, the detector performs $(1-2 \hat{d})=\operatorname{sgn}\left(\mathbf{c}^{H} \mathbf{r}\right)$, in which $\mathbf{c}$ is the weight vector used for combining and ${ }^{H}$ denotes conjugate transpose.

\section{Pulse Shaping}

According to the FCC UWB indoor emission limit [8], the -10dB-bandwidth must be kept within the band from 3.1 GHz to $10.6 \mathrm{GHz}$. However most UWB research [1], [2] has focused on systems where the received pulse is the second derivative of a Gaussian pulse, which does not meet the FCC UWB rules.

Here we define two pulse shapes also based on the Guassian pulse but can meet the FCC spectral requirements. We define the basic Guassian pulse in the time- and frequency-domain as:

$$
p_{0}\left(t, \tau_{m}\right)=\exp \left[-2 \pi\left(\frac{t}{\tau_{m}}\right)^{2}\right] \stackrel{F T}{\longleftrightarrow} P_{0}\left(f, \frac{1}{\tau_{m}}\right)=\frac{\sqrt{2} \tau_{m}}{2} \exp \left[-\frac{\pi}{2}\left(\tau_{m} f\right)^{2}\right]
$$

The $n^{\text {th }}$ derivative of $p_{0}\left(t, \tau_{m}\right)$ and the corresponding Fourier transform are 
represented by $p_{n}\left(t, \tau_{m}\right)=\left(\frac{d}{d t}\right)^{n} p_{0}\left(t, \tau_{m}\right) \quad$ and $\quad P_{n}\left(f, 1 / \tau_{m}\right)=(i 2 \pi f)^{n} P_{0}\left(f, 1 / \tau_{m}\right)$, respectively. By employing the $4^{\text {th }}$ derivative of $p_{0}\left(t, \tau_{m}\right)$, it turns out that we can meet the FCC UWB rules. For example with $p_{4}\left(t, \tau_{m}=0.17 \mathrm{~ns}\right)$ we can observe in Fig. 1(a), that the FCC UWB indoor emission limit [8] (plotted in Fig. 1(a) as a reference) is satisfied. The other approach is to shift the $2^{\text {nd }}$ derivative $p_{2}\left(t, \tau_{m}\right)$, by a carrier $f_{c}$, and take the upper sideband only. This results in another ultra-short pulse shape, $\tilde{p}_{2}\left(t, \tau_{m}, f_{c}\right)$, that is also shown in fig. 1(a) the case of $\tau_{m}=0.5 \mathrm{~ns}$ and $f_{c}=2.5 \mathrm{GHz}$, which complies with the FCC rules too. Time-domain waveforms for these two cases are shown in Fig. 1(b).

\section{THE EFFECT OF NBI ON UWB}

\section{A. Maximum Ratio Combining (MRC)}

A traditional ARake UWB receiver employs the weight vector $\mathbf{c}=\boldsymbol{\beta}$ to perform MRC, which maximizes the receiver output SNR (signal-to-noise ratio) when no interference exists. The receiver output SNR is given by:

$$
\operatorname{SNR}_{\text {out }}(\delta)=\frac{\left(A N_{s} m_{p}(\delta)\right)^{2}\left|\boldsymbol{\beta}^{H} \boldsymbol{\beta}\right|^{2}}{\boldsymbol{\beta}^{H} \mathbf{R}_{\mathbf{n}} \boldsymbol{\beta}}=\frac{N_{s}\left(A m_{p}(\delta)\right)^{2} \boldsymbol{\beta}^{H} \boldsymbol{\beta}}{\sigma_{r}^{2}}
$$

where, $\quad \boldsymbol{\beta}$ is the channel gain vector, and $\mathbf{R}_{\mathbf{n}}=E\left\{\mathbf{n} \mathbf{n}^{H}\right\}=N_{s} \sigma_{r}^{2} \boldsymbol{I}_{N_{r}}$ is a diagonal matrix of size $N_{r}$. However, in the presence of NBI, the noise samples are correlated and the choice of $\mathbf{c}=\boldsymbol{\beta}$ is no longer optimal. Assuming $d, \mathbf{i}$ and $\mathbf{n}$ mutually independent, the output SINR (signal-to-interference and noise ratio) is given by:

$$
\operatorname{SINR}_{\text {out }}(\delta)=\frac{\left(A N_{s} m_{p}(\delta)\right)^{2}\left|\boldsymbol{\beta}^{H} \boldsymbol{\beta}\right|^{2}}{\boldsymbol{\beta}^{H}\left(\mathbf{R}_{\mathbf{n}}+\mathbf{R}_{\mathbf{i}}\right) \boldsymbol{\beta}}
$$

where $\mathbf{R}_{\mathbf{i}}=E\left\{\mathbf{i i}^{H}\right\}$ is a $N_{r} \times N_{r}$ correlation matrix.

\section{B. Analysis of the Correlation Matrix $\mathbf{R}_{\mathbf{i}}=E\left\{\mathbf{i i}^{H}\right\}$}

As shown in (8), $\operatorname{SINR}_{\text {out }}(\delta)$ depends on $\mathbf{R}_{\mathbf{i}}=E\left\{\mathbf{i} \mathbf{i}^{H}\right\}$, i.e., the correlation matrix of the interference samples collected over a symbol interval at different paths. The $(k, l)$ element of $\mathbf{R}_{\mathbf{i}}$ is derived in Appendix I to have the following form:

$$
\left[\mathbf{R}_{\mathbf{i}}\right]_{k, l}=N_{s}\left|W_{r}\left(f_{I}\right)\right|^{2}\left[2 R_{\mathrm{i}}\left(\tau_{k}-\tau_{l}\right)-R_{\mathrm{i}}\left(\tau_{k}-\tau_{l}-\delta\right)-R_{\mathrm{i}}\left(\tau_{k}-\tau_{l}+\delta\right)\right]
$$


where, $f_{I}$ is the center frequency of the interference $\mathrm{i}(t), \tau_{k}$ and $\tau_{l}$ are the propagation delays for the $k^{\text {th }}$ and $l^{\text {th }}$ path, respectively, $R_{\mathrm{i}}(\tau)=\int_{-\infty}^{\infty} \mathrm{i}(t) \mathrm{i}^{*}(t+\tau) d t$ is the correlation function of $\mathrm{i}(t)$, and $W_{r}(f)=\int_{-\infty}^{\infty} w_{r}(t) e^{-j 2 \pi f t} d t$.

Each element of $\mathbf{R}_{\mathbf{i}}$ is proportional with $\left|W_{r}\left(f_{I}\right)\right|^{2}$, where $\left|W_{r}\left(f_{I}\right)\right|^{2}$ is the power spectral density of the received pulse $w_{r}(t)$ at the frequency $f_{I}$. This indicates that the effect of NBI on UWB systems is the severest when the NBI center frequency overlaps with the nominal center frequency of UWB spectrum. A better appreciation of $\mathbf{R}_{\mathbf{i}}$ can be obtained from specific models of the NBI.

\section{NBI Models}

We consider three models for the NBI and these consist of a single tone interferer, double-tone interferer and a general stochastic band-limited interferer. The first two models are defined respectively as

$$
\begin{gathered}
\mathrm{i}(t)=\alpha_{1} \sqrt{2 P_{1}} \cos \left(2 \pi f_{1} t+\phi_{1}\right) \\
\mathrm{i}(t)=\alpha_{1} \sqrt{2 P_{1}} \cos \left(2 \pi f_{1} t+\phi_{1}\right)+\alpha_{2} \sqrt{2 P_{2}} \cos \left(2 \pi f_{2} t+\phi_{2}\right)
\end{gathered}
$$

where $P_{1}, P_{2}$ and $f_{1}, f_{2}$ are the average powers and frequencies of the sinusoids, respectively, $\phi_{1}, \phi_{2}$ are two independent random phases, $\alpha_{1}, \alpha_{2}$ are independent complex channel gains. The third model can be considered as a zero-mean Gaussian random process with the following power spectral density (PSD):

$$
S_{\mathrm{i}}(f)= \begin{cases}P_{I} & f_{I}-\frac{B W}{2} \leq|f| \leq f_{I}+\frac{B W}{2} \\ 0 & \text { otherwise }\end{cases}
$$

where $f_{I}$ and $B W$ are the interference center frequency and bandwidth, respectively, and $P_{I}$ is the PSD of the interference within its bandwidth.

The corresponding correlation functions are, respectively:

$$
\begin{gathered}
R_{\mathrm{i}}(\tau)=P_{1}\left|\alpha_{1}\right|^{2} \cos \left(2 \pi f_{1} \tau\right) \\
R_{\mathrm{i}}(\tau)=\left|\alpha_{1}\right|^{2} P_{1} \cos \left(2 \pi f_{1} \tau\right)+\left|\alpha_{2}\right|^{2} P_{2} \cos \left(2 \pi f_{2} \tau\right) \\
R_{\mathrm{i}}(\tau)=2 P_{I} B W \cos \left(2 \pi f_{I} \tau\right) \operatorname{sinc}(B W \tau)
\end{gathered}
$$

The resultant $\mathbf{R}_{\mathbf{i}}$ 's are, respectively: 


$$
\begin{aligned}
{\left[\mathbf{R}_{\mathbf{i}}\right]_{k, l} } & =4 N_{s} P_{1}\left|\alpha_{1}\right|^{2}\left|W_{r}\left(f_{1}\right)\right|^{2}\left[\sin \left(\pi f_{1} \delta\right)\right]^{2} \cos \left(2 \pi f_{1}\left(\tau_{k}-\tau_{l}\right)\right) \\
{\left[\mathbf{R}_{\mathbf{i}}\right]_{k, l}=} & 2 N_{s}\left|W_{r}\left(f_{I}\right)\right|^{2} \sum_{j=1}^{2}\left|\alpha_{j}\right|^{2} P_{j}\left[\sin \left(\pi f_{j} \delta\right)\right]^{2} \cos \left(2 \pi f_{j}\left(\tau_{k}-\tau_{l}\right)\right) \\
{\left[\mathbf{R}_{\mathbf{i}}\right]_{k, l}=} & 2 N_{s} P_{I} B W\left|W_{r}\left(f_{I}\right)\right|^{2}\left[2 \cos \left(2 \pi f_{I}\left(\tau_{k}-\tau_{l}\right)\right) \operatorname{sinc}\left(B W\left(\tau_{k}-\tau_{l}\right)\right)\right. \\
& -\cos \left(2 \pi f_{I}\left(\tau_{k}-\tau_{l}-\delta\right)\right) \operatorname{sinc}\left(B W\left(\tau_{k}-\tau_{l}-\delta\right)\right) \\
& \left.-\cos \left(2 \pi f_{I}\left(\tau_{k}-\tau_{l}+\delta\right)\right) \operatorname{sinc}\left(B W\left(\tau_{k}-\tau_{l}+\delta\right)\right)\right]
\end{aligned}
$$

From equations (16)-(18) we can also observe that $\delta$ will have an impact on the effect of the NBI on UWB.

\section{NARROWBAND INTERFERENCE SUPPRESSION}

According to (9), (16)-(18) two possible solutions can be considered to suppress NBI. These are 1) $2 R_{\mathrm{i}}\left(\tau_{k}-\tau_{l}\right)-R_{\mathrm{i}}\left(\tau_{k}-\tau_{l}-\delta\right)-R_{\mathrm{i}}\left(\tau_{k}-\tau_{l}+\delta\right)=0$, and 2) $\left|W_{r}\left(f_{I}\right)\right|^{2}=0$, which can lead to $\left[\mathbf{R}_{\mathbf{i}}\right]_{k, l}=0$. These two solutions are investigated in the following along with a conventional MMSE combining approach.

\section{A. NBI Suppression Employing the BPPM Modulation Parameter $\delta$}

The realization of the first solution depends on the correlation function $R_{\mathrm{i}}(\tau)$ of the interference $\mathrm{i}(t)$ as well as the BPPM modulation parameter $\delta$. For a single tone interferer (see (10), (13), (16)) we can immediately observe that $\left[\mathbf{R}_{\mathbf{i}}\right]_{k, l}=0$ when $f_{1} \cdot \delta=l(l=1,2, \cdots)$ and therefore perfect interference suppression can be achieved by letting $\delta=1 / f_{1}, 0<\delta<T_{w}$, where $T_{w}$ is the pulse duration. However when NBI consists of two or more carriers (see (11), (14), (17)), perfect suppression cannot be achieved. But if we let $f_{I}=\left(f_{1}+f_{2}\right) / 2$ represent the center frequency of the interference and $B W=\left|f_{1}-f_{2}\right|$ the nominal bandwidth, when $B W \ll f_{I}$, $f_{j} \delta=\left(f_{I} \pm B W / 2\right) \delta \approx f_{I} \delta \quad(j=1,2)$, we can still eliminate some NBI by setting $\delta=1 / f_{I}, 0<\delta<T_{w}$. For the third NBI model (see (12), (15), (18)), when $B W \ll f_{I} \quad$ and $\quad \delta=1 / f_{I} \quad, \quad \cos \left(2 \pi f_{I}\left(\tau_{k}-\tau_{l} \pm \delta\right)\right)=\cos \left(2 \pi f_{I}\left(\tau_{k}-\tau_{l}\right)\right) \quad$ and $\operatorname{sinc}\left(B W\left(\tau_{k}-\tau_{l} \pm \delta\right)\right) \approx \operatorname{sinc}\left(B W\left(\tau_{k}-\tau_{l}\right)\right)$, which also lead to $\left[\mathbf{R}_{\mathbf{i}}\right]_{k, l} \approx 0$.

The above analysis shows that, under the condition of $B W \ll f_{I}, \mathrm{NBI}$ suppression can be achieved by simply setting the BPPM modulation parameter $\delta=1 / f_{I}, 0<\delta<T_{w}$. When $1 / f_{I} \geq T_{w}$, this scheme cannot be performed and the 
value of $\delta$ is chosen to maximize $m_{p}(\delta)$.

\section{B. Notch Filtering in the Frequency Domain}

To obtain the second solution, i.e., $\left|W_{r}\left(f_{I}\right)\right|^{2}=0$, it is necessary to notch out the part of the power spectrum $\left|W_{r}(f)\right|^{2}$ that is around the frequency $f_{I}$. To make the theoretical analysis straightforward, the discussion that follows is limited to the signal received during the $i^{\text {th }}$ pulse-repetition interval $\left(i T_{f} \leq t \leq(i+1) T_{f}\right)$ :

$$
\mathrm{r}(t)=\beta_{k} A w_{r}\left(t-i T_{f}-c_{i} T_{c}-\delta d-\tau_{k}\right)+\mathrm{i}(t)+\mathrm{n}(t)
$$

First, the Fourier transform of (19) is taken. Then the transform is multiplied by the transfer function of the notch filter, whose transfer function and impulse response are defined, respectively, as:

$$
\begin{gathered}
H(f)=1-\left[u\left(f-f_{I}+\frac{B W_{H}}{2}\right)-u\left(f-f_{I}-\frac{B W_{H}}{2}\right)\right] \\
h(t)=\delta(t)-B W_{H} e^{j 2 \pi f_{I} t} \operatorname{sinc}\left(B W_{H} t\right)
\end{gathered}
$$

where, $B W_{H}$ represents the width of the spectral notch, which is set to be sufficiently larger than the bandwidth of the interference. In practice this process could be performed with a filter bank.

Having notched out the interference, the remaining signal is restored to the time domain by performing inverse Fourier transform, which can be expressed as:

$$
\begin{aligned}
& \widetilde{\mathrm{r}}(t)=\beta_{k} A w_{\text {notch }}\left(t-i T_{f}-c_{i} T_{c}-\delta d-\tau_{k}\right)+\mathrm{n}(t) \\
& w_{\text {notch }}(t)=w_{r}(t)-W_{r}\left(f_{I}\right) B W_{H} e^{j 2 \pi f_{I} t} \operatorname{sinc}\left(B W_{H} t\right)
\end{aligned}
$$

Note that in (23), $t$ is in nanoseconds and $B W_{H}$ is in GHz. For suppression of typical NBI, $B W_{H}<<1$; moreover, due to the low transmission power of UWB systems, $W_{r}\left(f_{I}\right)$ is also of a small value. So $w_{\text {notch }}(t)$ is expected to be only slightly different from $w_{r}(t)$. The rest of the signal processing takes place in time domain.

\section{Minimum Mean-Square-Error Combining (MMSEC)}

In the presence of NBI, the optimal weigh vector c used for combining at the Rake receiver, is the one that minimize the following Mean-Square-Error (MSE):

$$
M S E=E\left\{\left[\mathbf{c}^{H} \mathbf{r}-(1-2 d) A N_{s} m_{p}(\delta)\right]^{2}\right\}
$$

Simple calculation yields that the Minimum-MSE weight vector has the form as: 


$$
\mathbf{c}=\frac{\left(A N_{s} m_{p}(\delta)\right)^{2}\left(\mathbf{R}_{\mathbf{i}}+\mathbf{R}_{\mathbf{n}}\right)^{-1} \boldsymbol{\beta}}{1+\left(A N_{s} m_{p}(\delta)\right)^{2} \boldsymbol{\beta}^{H}\left(\mathbf{R}_{\mathbf{i}}+\mathbf{R}_{\mathbf{n}}\right)^{-1} \boldsymbol{\beta}}
$$

The MMSE combining approach is to employ the MMSE weight vector (25) to perform $(1-2 \hat{d})=\operatorname{sgn}\left(\mathbf{c}^{H} \mathbf{r}\right)$ at the Rake receiver. According to the expression (4) for $\mathbf{r}$, it is easily seen that this MMSE combining is performed every symbol. The resulting maximal output SINR, denoted by $\operatorname{SINR}_{M M S E}$, is

$$
\operatorname{SINR}_{M M S E}=\left(A N_{s} m_{p}(\delta)\right)^{2} \boldsymbol{\beta}^{H}\left(\mathbf{R}_{\mathbf{i}}+\mathbf{R}_{\mathbf{n}}\right)^{-1} \boldsymbol{\beta}
$$

\section{PERFORMANCE EVALUATION}

The effect of NBI on UWB TH systems and the single-link performance of the proposed NBI suppression schemes were evaluated via two performance measures: average output SINR and average bit error rate (BER). By "average", we mean that these two measures are obtained by averaging over different channel gains and delays. The numerical results assumed the pulse $p_{4}\left(t, \tau_{m}=0.17 \mathrm{~ns}\right)$ in Fig. $1(\mathrm{~b})$ as the received pulse. The indoor channel model described in [6] was used. Without loss of generality, we assumed $E\left\{\boldsymbol{\beta}^{H} \boldsymbol{\beta}\right\}=1, R_{s}=1 \mathrm{Mb} / \mathrm{s}$, and $T_{f}=100 \mathrm{~ns}$. In all the results the interference-to-signal ratio (ISR) per pulse was $30 \mathrm{~dB}$, the SNR per pulse was $0 \mathrm{~dB}$, and the notch width of the spectral notch was fixed at $40 \mathrm{MHz}$.

The average output SINR for the cases of single-tone NBI and band-limited NBI is depicted, as a function of the interference center frequency $f_{I}$, in Fig. 2, and Fig. 3 respectively. Results are provided for both the "ARake" (All-Rake) and the "PRake" (Partial-Rake) with the first " $L_{p}$ " paths. Here we choose $L_{p}=16$ according to the PRake performance evaluation in [7] that was also based on the UWB channel model [6], in which the results showed that the performance of a PRake with more than 16 fingers is reasonably close to the ARake. For each Rake configuration, results for "MRC" and our three suppression schemes, "MMSE" for MMSE Combining, "delta" for the scheme that employs the modulation parameter $\delta$, and "notch" for frequency-domain notch filtering, are shown. The corresponding average BER vs. $f_{I}$ for the case of single-tone NBI is plotted in Fig. 4. By comparing the "MRC" curves in Fig. 2 - Fig. 4 with the power spectrum of $p_{4}\left(t, \tau_{m}=0.17 \mathrm{~ns}\right)$ in Fig. 1(a), we 
observe that the performance degradation is the highest when the nominal center frequencies for NBI and UWB spectrum are overlapping as shown in Section III. The numerical results also reveal that for an indoor channel with large number of paths, all the proposed NBI rejection schemes in conjunction with an ARake can achieve a near-absolute NBI suppression. The simpler PRake with 16 fingers can provide performance comparable to that of the ARake. There is a trade-off however in that reduced receiver complexity reduces performance.

As for which scheme to use in a given scenario, it appears that each one has its own applicability. Both the "delta" scheme and frequency-domain notching need to estimate the interference frequency, but not MMSE Combining. The "delta" scheme can annul only NBI with center frequency above $1 / T_{w}$. The spectral notch method is simple, but complete annulment will not be possible because of non-ideal filters. The center frequency of the notch will also need to be adjustable to meet the specific NBI.

In Fig. 5 we show the average BER vs. SNR per pulse for each Rake configuration with MRC and the three NBI suppression schemes, when the interference is from an OFDM-based WLAN device with BPSK modulation. The OFDM carrier frequency is set at $5.2 \mathrm{GHz}, 52 \mathrm{sub}$-carriers are used, which result in a bandwidth of $16.56 \mathrm{MHz}$, and the transmission from the interferer is assumed continuative. As with the other simulations an ISR of $30 \mathrm{~dB}$ is used. Again significant performance loss occurs when NBI suppression techniques are not applied.

\section{CONCLUSIONS}

In this letter we demonstrate the effect of NBI on the performance of UWB TH receivers. The results demonstrate that significant performance loss is possible when the NBI is of sufficient power. For example when using UWB pulse shapes that meet the FCC Part 15 rules, interference from a nearby IEEE 802.11a device will affect UWB performance if the ISR per pulse is greater than $30 \mathrm{~dB}$. We also investigate the performance of three schemes to mitigate the effect of the NBI. These allow the effects of NBI to be nearly eliminated but each approach has different tradeoffs in terms of complexity and performance. 


\section{APPENDIX I}

The expression for the $(k, l)$ element of $\mathbf{R}_{\mathbf{i}}=E\left\{\mathbf{i} \mathbf{i}^{H}\right\}$ is developed as follows:

$$
\begin{aligned}
{\left[\mathbf{R}_{\mathrm{i}}\right]_{k, l}=} & E\left\{\mathrm{i}_{k} \mathrm{i}_{l}^{*}\right\} \\
= & \sum_{i=0}^{N_{s}-1} \sum_{j=0}^{N_{s}-1} \int_{-\infty}^{\infty} \int_{-\infty}^{\infty} E\left\{\mathrm{i}\left(t+i T_{f}+c_{i} T_{c}+\tau_{k}\right) \mathrm{i}^{*}\left(\tau+j T_{f}+c_{j} T_{c}+\tau_{l}\right)\right\} \mathcal{N}(t) v(\tau) d t d \tau \\
\approx & N_{s} \int_{-\infty}^{\infty} \int_{-\infty}^{\infty} R_{\mathrm{i}}\left(t-\tau+\tau_{k}-\tau_{l}\right)\left[w_{r}(t)-w_{r}(t-\delta)\right]\left[w_{r}(\tau)-w_{r}(\tau-\delta)\right] d t d \tau \\
= & N_{s}\left[2 \int_{-\infty}^{\infty} \int_{-\infty}^{\infty} R_{\mathrm{i}}\left(t-\tau+\tau_{k}-\tau_{l}\right) w_{r}(t) w_{r}(\tau) d t d \tau\right. \\
& -\int_{-\infty}^{\infty} \int_{-\infty}^{\infty} R_{\mathrm{i}}\left(t-\tau+\tau_{k}-\tau_{l}-\delta\right) w_{r}(t) w_{r}(\tau) d t d \tau \\
& \left.-\int_{-\infty}^{\infty} \int_{-\infty}^{\infty} R_{\mathrm{i}}\left(t-\tau+\tau_{k}-\tau_{l}+\delta\right) w_{r}(t) w_{r}(\tau) d t d \tau\right]
\end{aligned}
$$

Since the UWB spectrum will be basically constant over the frequency range of NBI that centered at $f_{I}$, the above expression can be approximated as:

$$
\left[\mathbf{R}_{\mathrm{i}}\right]_{k, l}=N_{s}\left|W_{r}\left(f_{I}\right)\right|^{2}\left[2 R_{\mathrm{i}}\left(\tau_{k}-\tau_{l}\right)-R_{\mathrm{i}}\left(\tau_{k}-\tau_{l}-\delta\right)-R_{\mathrm{i}}\left(\tau_{k}-\tau_{l}+\delta\right)\right]
$$

\section{REFERENCES}

[1] M. Z. Win, R. A. Scholtz, "Ultra-wide bandwidth time-hopping spread-spectrum impulse radio for wireless multiple-access communications," IEEE Trans. Commun., vol. 48, pp. 679-689, April 2000.

[2] I. Bergel, E. Fishler, H. Messer, "Narrow-band interference suppression in time-hopping impulse-radio systems," IEEE Conf. UWB Systems and Tech., 2002, pp. 303-308.

[3] Z. Li, A. M. Haimovich, H. Grebel, "Performance of ultra-wideband communications in the presence of interference," IEEE Int. Conf. Communications, vol. 10, June 2001, pp. 2948-2952.

[4] M. S. Iacobucci, A. Di Benedetto, L. De Nardis, "Radio frequency interference issues in impulse radio multiple access communication systems," IEEE Conf. UWB Systems and Tech., 2002, pp. 293-296.

[5] M. HainaIainen, R. Tesi, J. Iinatti, "On the UWB system performance studies in AWGN channel with interference in UMTS band," IEEE Conf. UWB Systems and Tech., 2002, pp. 321-326.

[6] D. Cassioli, M. Z. Win, A. F. Molisch, “A statistical model for the UWB indoor channel," IEEE Vehic. Tech. Conf., vol. 2, Rhodos, Greece, May 2001, pp. 1159-1163.

[7] D. Cassioli, M. Z. Win, F. Vatalaro, A. F. Molisch, "Performance of low-complexity rake reception in a realistic UWB channel," IEEE Int. Conf. Communications, vol. 2, April 2002, pp. 763-767.

[8] "New public safety applications and broadband internet access among uses envisioned by FCC authorization of Ultra-Wideband technology," Feb. 14, 2002.

http://www.fcc.gov/Bureaus/Engineering_Technology/News_Releases/2002/nret0203.ppt 


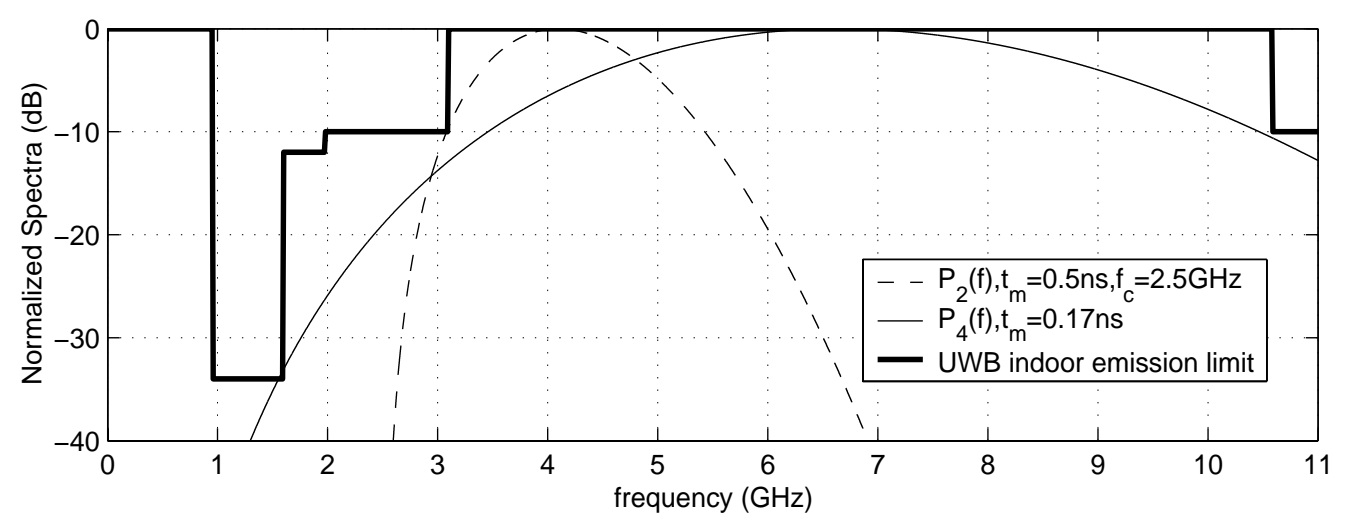

(a)

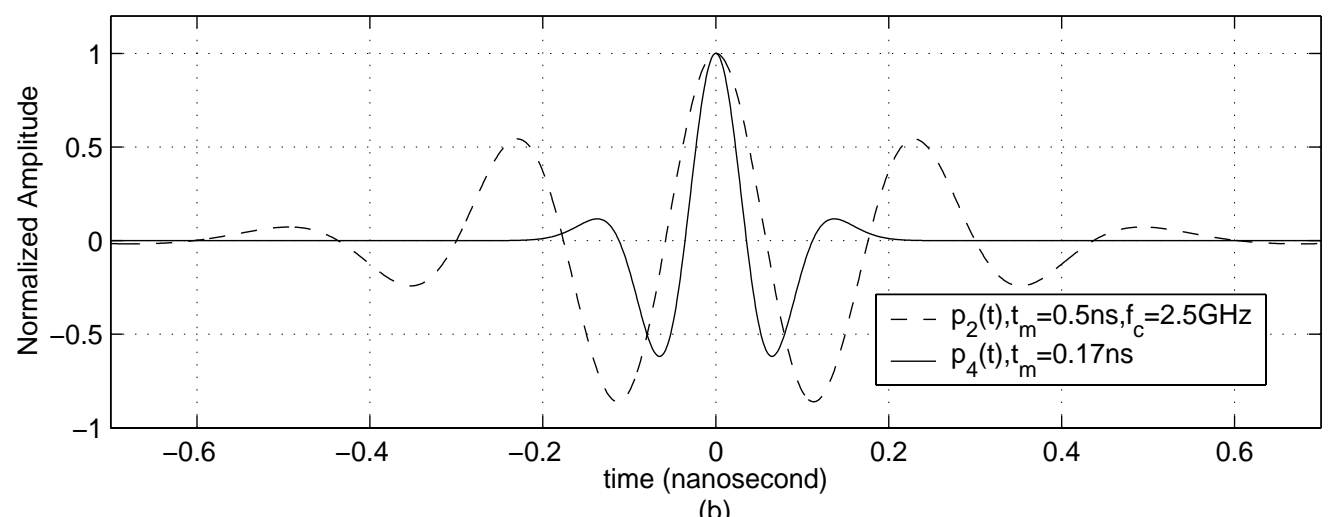

(b)

Fig. 1. (a) Power spectra of the two pulses proposed in Part D, Section II, and FCC preliminary UWB emission limit for indoor systems. (b) The corresponding waveforms of the two proposed pulses as functions of time in nanoseconds. 


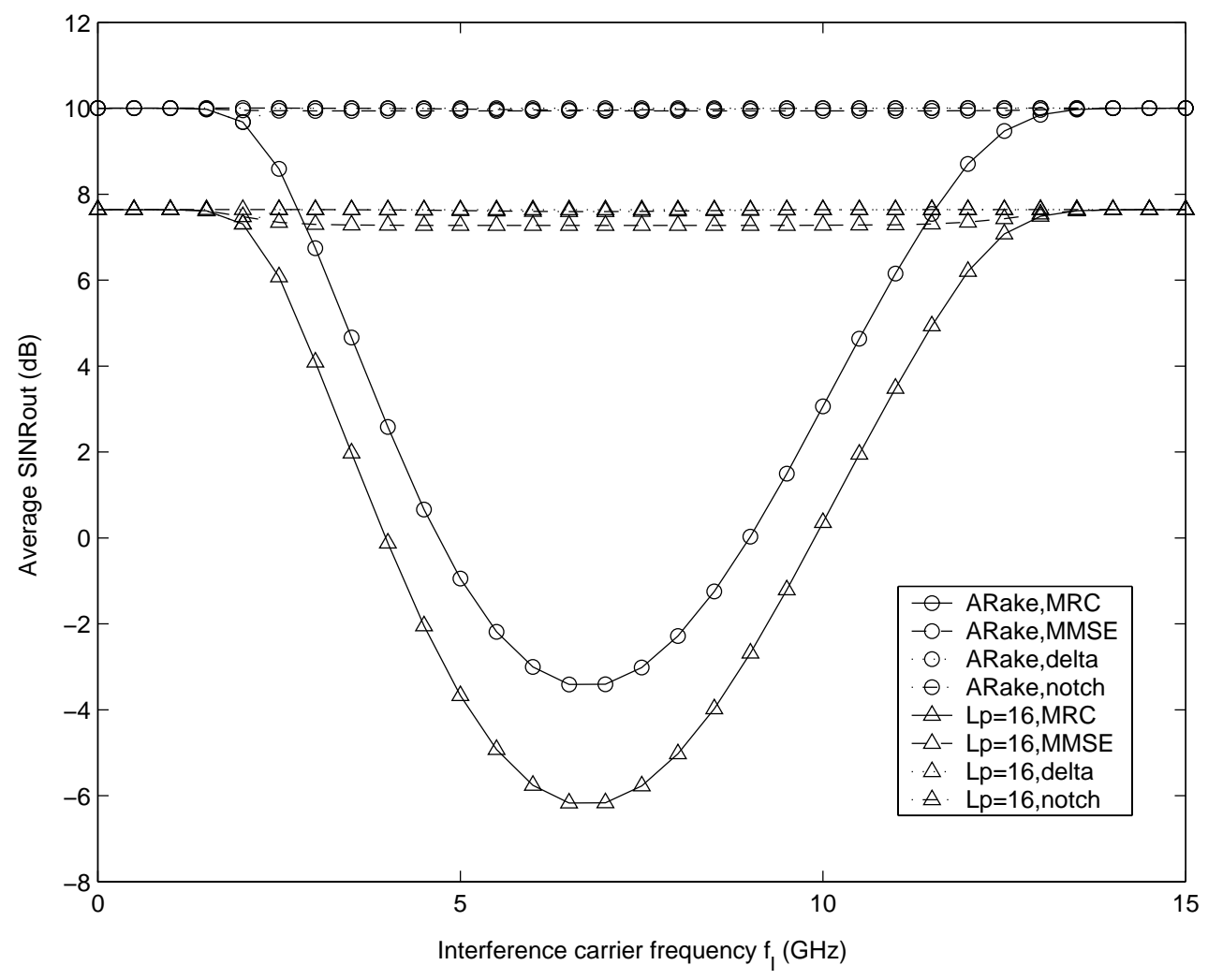

Fig. 2. The average output SINR vs. the interference carrier frequency $f_{I}$ for the case of single-tone NBI. 


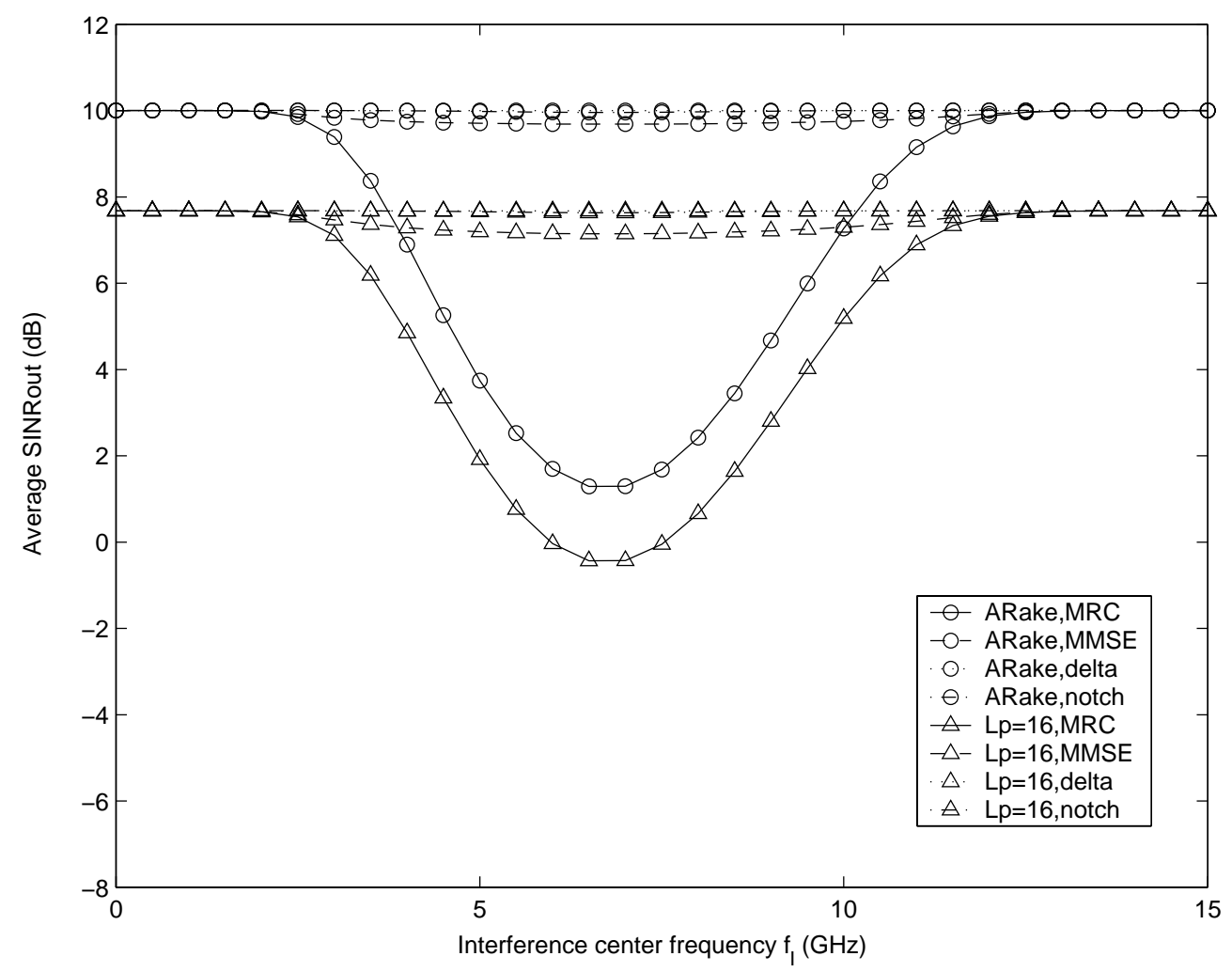

Fig. 3. The average output SINR vs. the interference center frequency $f_{I}$ for the case of NBI with a constant spectrum over a bandwidth of $20 \mathrm{MHz}$. 


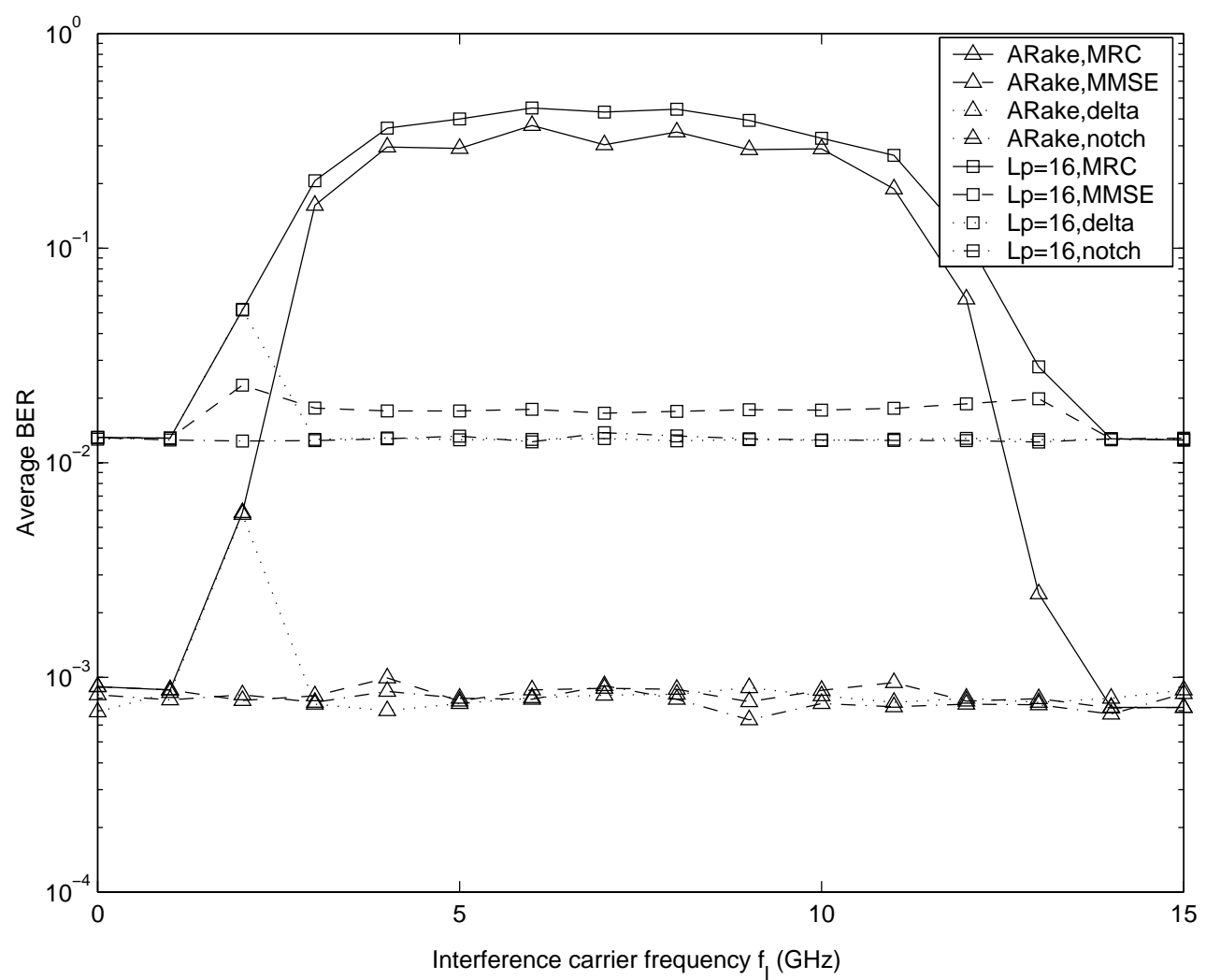

Fig. 4. The average BER vs. the interference carrier frequency $f_{I}$ for the case of single-tone NBI. 


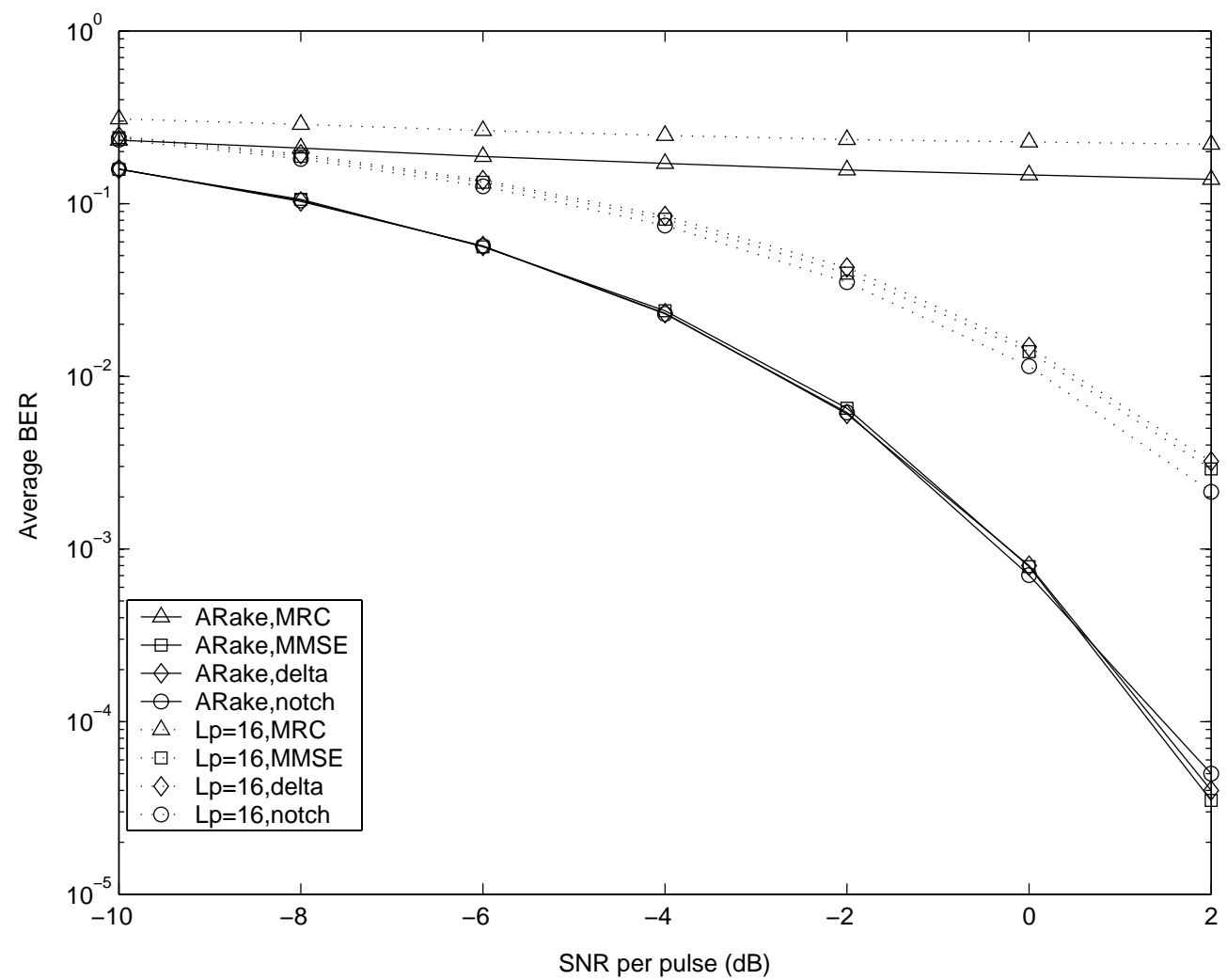

Fig. 5. The average BER vs. SNR per pulse for the case of NBI caused by an OFDM-based WLAN signal. The OFDM carrier frequency is set at $5.2 \mathrm{GHz}$. 52 subcarriers are used, which result in a bandwidth of $16.56 \mathrm{MHz}$. 